\title{
Deformation Behavior of Cross-Linked Supercrystalline Nanocomposites: An in Situ SAXS/WAXS Study during Uniaxial Compression
}

\author{
Diletta Giuntini,* Anton Davydok, Malte Blankenburg, Berta Domènech, Büsra Bor, Mingjing Li, \\ Ingo Scheider, Christina Krywka, Martin Müller, and Gerold A. Schneider
}

Cite This: Nano Lett. 2021, 21, 2891-2897

Read Online

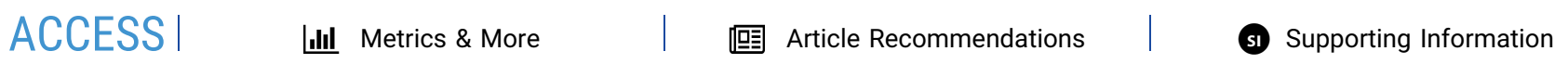

ABSTRACT: With the ever-expanding functional applications of supercrystalline nanocomposites (a relatively new category of materials consisting of organically functionalized nanoparticles arranged into periodic structures), it becomes necessary to ensure their structural stability and understand their deformation and failure mechanisms. Inducing the cross-linking of the functionalizing organic ligands, for instance, leads to a remarkable enhancement of the nanocomposites' mechanical properties. It is however still unknown how the cross-linked organic phase redistributes applied loads, how the supercrystalline lattice accommodates the imposed deformations, and thus in general what phenomena

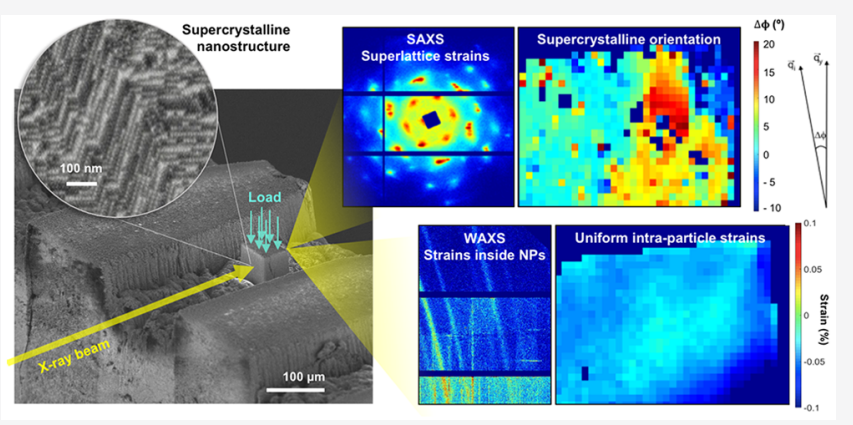
govern the overall material's mechanical response. This work elucidates these aspects for cross-linked supercrystalline nanocomposites through an in situ small- and wide-angle X-ray scattering study combined with uniaxial pressing. Because of this loading condition, it emerges that the cross-linked ligands effectively carry and distribute loads homogeneously throughout the nanocomposites, while the superlattice deforms via rotation, slip, and local defects generation.

KEYWORDS: Supercrystals, Nanocomposites, Mechanical Behavior, X-ray Scattering, Cross-Linking, Nanocrystal Superlattice

Cupe upercrystalline nanocomposites are a rising category of hybrid inorganic-organic materials. They generally consist of inorganic nanoparticles, surface-functionalized with organic ligands and organized into periodic close-packed structures, known as superlattices, in analogy with crystalline lattices. This combination of nanosized building blocks and their arrangement into superlattices leads to a variety of emergent collective properties and in turn to a diverse set of applications in optoelectronics, energy, catalysis, sensors, and magnetic devices. $^{1-8}$ Supercrystalline nanocomposites also show great promise as structural materials, in connection with the field of biomimicry. Biomaterials feature impressive combinations of mechanical properties because of their internal architecture, characterized by a high concentration of mineral nanoparticles (NPs) in an ultrathin organic matrix, design principles that supercrystals also feature. ${ }^{3,9}$ Understanding, enhancing, and better controlling the mechanical behavior of supercrystalline nanocomposites are thus needed to widen their functional applications and allow integration into robust devices. ${ }^{10}$

The assessment of supercrystals' mechanical properties has initially focused on ultrathin films ${ }^{11,12}$ and later included 3D single supercrystals. ${ }^{13-15}$ The characteristic parameters of NPs (size, shape, faceting), functionalizing ligands (chemical structure, length, grafting density, configuration), and meso- scale arrangement (periodicity, polymorphism, interparticle distances) all play important roles, sometimes with multifold implications. ${ }^{16,17}$ The elastic modulus and hardness compare to the ones of hard polymers, a remarkable outcome for material systems mainly held together by van der Waals interactions but still insufficient for structural applications (such as bioimplants) also because of the low fracture toughness $\left(\sim 40 \mathrm{kPa} \cdot \mathrm{m}^{1 / 2}\right){ }^{15}$

An important step forward toward strengthening supercrystalline nanocomposites has been found in inducing the cross-linking of the organic ligands via annealing at moderate temperatures $\left(250-350{ }^{\circ} \mathrm{C}\right) .^{18-26}$ The presence of covalent bonds among neighboring ligand molecules, which are in turn anchored to the NPs, becomes the dominating factor holding the supercrystals together. Nanoindentation has shown that elastic modulus and hardness shift from $\sim 15$ to $\sim 64 \mathrm{GPa}$ and

Received: December 22, 2020

Revised: March 17, 2021

Published: March 22, 2021 

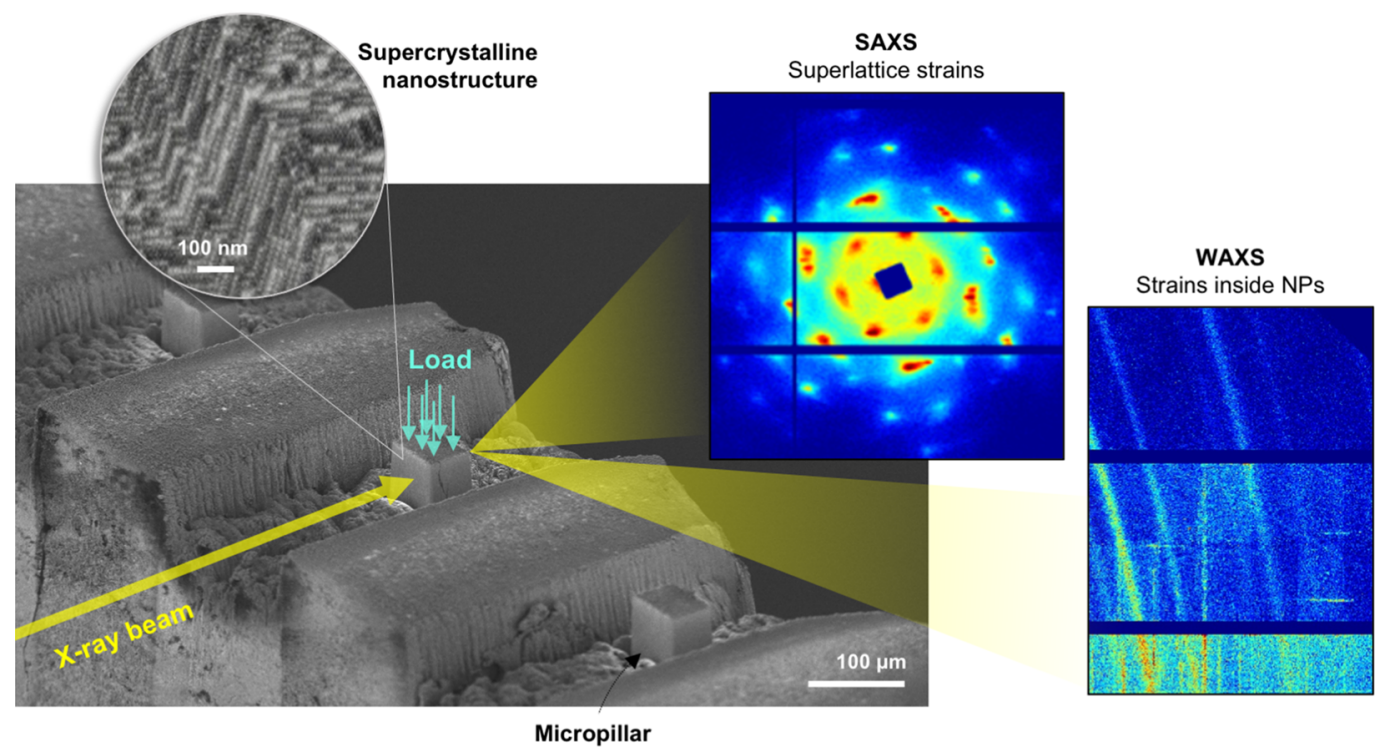

Figure 1. In situ X-ray scattering-microcompression testing setup. Supercrystalline nanocomposite micropillars are uniaxially compressed with a uniform load (through a flat diamond punch) while gathering small- and wide-angle X-ray scattering signals (SAXS and WAXS). The pillars were scanned edge to edge and from the top for a downward extension of $30 \mu \mathrm{m}$, with a microfocused beam to maximize resolution. SAXS provides insights on the superlattice deformations (order of tens of nm) and WAXS on the strains in the NPs' crystalline lattice (order of $\AA$ ).

from $\sim 0.6$ to $\sim 4.7 \mathrm{GPa}$, respectively, between non-cross-linked and cross-linked materials. ${ }^{19}$ The degree of cross-linking also plays a role, with compressive and bending strengths increasing from $\sim 440$ to $\sim 740 \mathrm{MPa}$ and from $\sim 210$ to $\sim 460 \mathrm{MPa}$, respectively, when annealing the material at 250 or $350{ }^{\circ} \mathrm{C} .{ }^{18}$

Questions are however still open on the role of the crosslinked organic phase in the overall material's behavior under loading. It has not yet been elucidated whether such a strengthening effect is only due to the cross-linked phase or if local direct particle-particle contacts are present and thus responsible for the load-carrying capability of the nanocomposites (the ligand coverage on the NPs could be nonuniform). It is also still unclear how the superlattice responds to external loads, namely, what strains it can accommodate and what dominates its deformation behavior, e.g., the NP arrangement in tightly packed structures or the mechanical properties of the constituent materials.

A tailored experiment has therefore been designed to answer all these questions simultaneously, bridging the atomic scale and the nanocomposites' mesoscale. It consists of the combination of a supercrystalline micropillar's uniaxial compression with wide- and small-angle X-ray scattering (WAXS/SAXS), which simultaneously monitors the alterations of the NPs' crystalline lattice and of the nanocomposites' superlattice.

In situ X-ray scattering has been applied to nanocrystal superlattices for different purposes, starting with the kinetics of phase transformations within nanocrystals, ${ }^{27}$ followed, with the rise of supercrystals, by SAXS studies monitoring superstructures nucleation and growth ${ }^{28}$ and later by investigations on processing-induced strains (also via Raman spectroscopy ${ }^{29}$ ) and on superlattice stability under increasing temperatures. ${ }^{30}$ When it comes to mechanical loading, deformations in supercrystals have been explored in hydrostatic pressing conditions, from which it has emerged that they feature high structural stability and compressibility. ${ }^{31-39}$ Hydrostatic compression can also lead to improved supercrystallinity or, in the presence of a deviatoric stress, to sintering of the NPs.
These variations are traced back to the role played by the organic ligands, acting as interparticle spacers, and by the superstructure parameters, as molecular dynamics simulations confirm. ${ }^{40,41}$

Cross-linked supercrystalline nanocomposites are expected to feature a behavior that largely deviates from the cases above. In a cross-linked phase the ligands cannot easily migrate nor can the NPs be easily rearranged in the superlattice. This study elucidates the load-carrying and deformation mechanisms of cross-linking-strengthened supercrystalline nanocomposites, for loading conditions that allow shear deformations, which are a critical material failure mechanism likely to occur in many applications (such as wearable and flexible devices).

Large-size $\left(>2 \times 10^{5} \mu \mathrm{m}^{3}\right)$ pillars were tested under uniaxial compression (the pillars had free lateral surfaces). Under this uniform loading condition, it is possible to detect both the presence of load percolation paths at direct particle-particle contacts and shear deformations at the mesoscale (superlattice). The first question to be answered is whether the mechanical response of the cross-linked nanocomposites is governed by the cross-linked ligands, forming a uniform ultrathin interphase and distributing the load equally on all NPs. If, alternatively, the ligands do not form a uniform interphase, localized direct contacts between the inorganic NP cores will occur. These contacts could then form load percolation paths, while the rest of the material would stay relatively unstressed. Load percolation paths would lead to local strains at the inter-NP contacts, resulting in alterations of the lattice constant of the NP crystalline structure, detectable with wide-angle X-ray scattering (WAXS, which can detect the lattice parameters of the order of $\AA$ and their variation in atomic crystals). The second question concerns the deformation mechanisms at the superlattice scale and specifically whether the supercrystals accommodate the imposed deformation by straining (superlattice constant alterations), rotation, slip, or additional defects formation. These kinds of superlattice alterations can be detected via small-angle X-ray 
scattering (SAXS), a technique sensitive to larger lattice parameters, like the tens of nm characteristic of supercrystals.

The in situ microfocus X-ray scattering study during uniaxial compression was conducted at the Nanofocus Endstation (NFE) of the P03 beamline at the PETRA III Synchrotron in DESY (Hamburg, Germany). Pillars with size $\sim 50 \times 70 \times 60$ $\mu \mathrm{m}^{3}$ were produced via laser cutting and plasma focused ion beam (plasma-FIB) in bulk iron oxide $\left(\mathrm{Fe}_{3} \mathrm{O}_{4}\right)$-oleic acid supercrystalline nanocomposites with cross-linked organic ligands. The nanocomposites ( $\mathrm{mm}$ size) were obtained from an initial suspension of the functionalized NPs (radius of the inorganic core $R=7.9 \pm 1.3 \mathrm{~nm}$; see Supporting Information section 1 and Figure S1) with a three-step routine (reported elsewhere in detail ${ }^{19}$ and summarized in the Supporting Information, Methods), consisting of self-assembly via solvent destabilization, pressing, and heat treatment at $325^{\circ} \mathrm{C}$ in inert atmosphere, the latter inducing the ligands' cross-linking. The obtained $\mathrm{cm}$-sized nanocomposites are polysupercrystalline, and therefore the tested micropillars can contain multiple supercrystalline domains ("grains"). The typical domain size is in the order of tens of $\mu \mathrm{m}$, and thus the tested micropillars contain on average 1-2 domains. The micropillars were compressed with a flat punch (Synton-MDP LTD, Switzerland) in a self-developed indenter available at P03's NFE, ${ }^{42}$ while X-ray scattering signals were collected. Synchrotron radiation was chosen so that a microfocused beam could be used to scan supercrystalline pillars extending several tens of $\mu \mathrm{m}$ while preserving a high resolution. Figure 1 illustrates the testing setup and examples of SAXS and WAXS patterns.

The compression proceeded in displacement-control mode, by pressing the micropillars against the fixed flat punch until fracture. Simultaneous SAXS and WAXS scans were taken at multiple compression stages under constant load (see Supporting Information, Methods). The resulting forcedisplacement curves are shown in Figure 2. Two micropillars were pressed during preparatory tests, and the third ("main") pillar is the one to which the following X-ray analysis refers (see Supporting Information section 2 and Figure S2). The displacements are corrected for the influence of the indentation of the pillars into the surrounding substrate (see

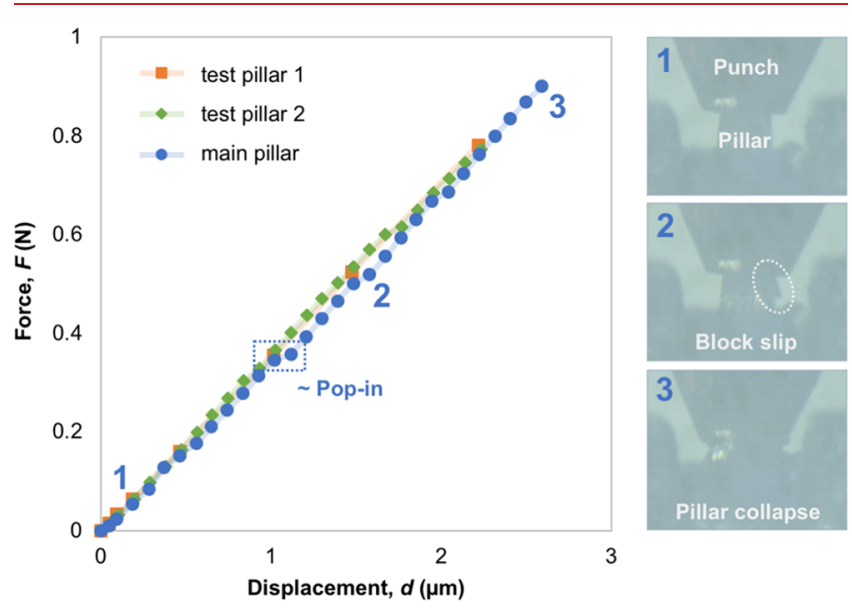

Figure 2. Force-displacement $(F-d)$ curves of the tested micropillars. All pillars showed reproducible linear elastic behavior. Sudden changes of slope (slight pop-in phenomena) are associated with material slip. Optical micrographs taken in the beam direction in between X-ray scans of the main pillar show selected stages of the compression tests: (1) contact, (2) onset of block slip, (3) failure.
Supporting Information section 2 and Figure S3 for details and stress-strain data). A linear elastic behavior emerges, with remarkable repeatability among the different micropillars. Final failure occurred in the form of a brittle fracture, immediately anticipated by buckling of the micropillars. In one case (main pillar), a slip of a block of material was observed instead of buckling, as highlighted in Figure 2 and Figure S4. We assume that slip is responsible for the small pop-in-like steps, sudden slope changes indicating displacement bursts. It is likely because of this gradual slip accommodating the load that this pillar withstands higher load values $(0.90 \mathrm{~N}$, i.e., $264 \mathrm{MPa}$ strength) with respect to the other two ( 0.78 and $0.76 \mathrm{~N}$, i.e., 233 and $222 \mathrm{MPa}$ ). A previous numerical study has shown that cross-linked iron oxide-oleic acid supercrystalline nanocomposites have a Poisson's ratio $\nu=0.34 .^{22}$ Given the average strain to failure of the pillars, $3.8 \%$, this implies a transversal strain of $\sim 1.3 \%$. Note that the resulting average compressive strength and elastic modulus (239 $\mathrm{MPa}$ and 6.4 GPa; see Supporting Information section 2) are lower than the ones measured ex situ in the same kind of material (1.2 and 47 $\mathrm{GPa}$ ). This is not unexpected and can be explained by several factors (Supporting Information section 3 and Figure S5), such as nonstandard testing conditions for the elastic modulus, and the well-known size effect for strength. ${ }^{18,29,43-46}$ The potential load relaxation during the holding times necessary for the $\mathrm{X}$ ray measurements ( $\sim 40 \mathrm{~min}$ for a whole scan) was also evaluated. A decrease in the measured load between the start and the end of the scan was detected, more significant at the earliest stage of compression (up to $40 \%$, i.e., a decrease from 9 to $5 \mathrm{mN}$ in the first compression step) and negligible ( $5 \%)$ afterward. Viscosity can play a role in materials containing an organic phase, even though preliminary data from a follow-up work indicate that creep effects are negligible in this study. ${ }^{47} \mathrm{X}$ ray measurements were performed at a higher frequency during the first test stages (up to $\sim 0.2 \mu \mathrm{m}, 10 \%$ of the total deformation), but no significant difference was nevertheless observed in the predominantly linear elastic behavior of the nanocomposites, as ex situ tests confirm (Supporting Information section 3).

We first look into the load distribution within the micropillar through the strains experienced by the NPs, i.e., through WAXS. Each iron oxide NP is a single nanocrystal of $\mathrm{Fe}_{3} \mathrm{O}_{4}$ (magnetite), even if previous work has revealed that traces of $\gamma-\mathrm{Fe}_{2} \mathrm{O}_{3}$ (maghemite) can be present. ${ }^{21}$ Both phases have inverse spinel structure with almost coincident lattice parameters $\left(a=0.839 \mathrm{~nm}\right.$ for $\mathrm{Fe}_{3} \mathrm{O}_{4}$ and $a$ in the range of 0.834-0.839 nm for $\left.\gamma-\mathrm{Fe}_{2} \mathrm{O}_{3}\right)^{48}$ and therefore equivalent behavior for the purposes of this study. Figure 3A shows a representative frame of the short distance (WAXS) detector with continuous high-order Debye-Scherrer rings (each NP is randomly oriented within the superlattice). After radial integration of the WAXS spectra, the most intense and position-sensitive peak was identified, via Gaussian fit, for $q=$ $47.5 \mathrm{~nm}^{-1}$. On the basis of this peak, strain maps relative to the NPs' crystalline lattice were obtained for the different compression stages, shown in Figure 3B. A preloading scan of the micropillar was used as reference (zero strain). Due to experiment geometry and vacuum path required for SAXS measurements, the WAXS detector was inclined $\sim 7^{\circ}$ with respect to the beam direction, and thus the plots in Figure $3 \mathrm{~B}$ show this projection on the detector plane.

A largely uniform strain field emerges. The diagonally oriented nonuniformities, indicating larger strains in the 


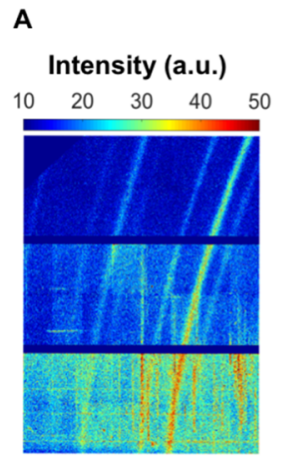

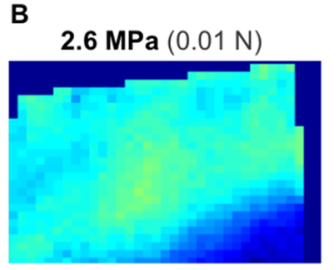

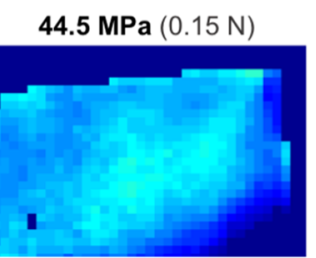

6.7 MPa $(0.02 \mathrm{~N})$

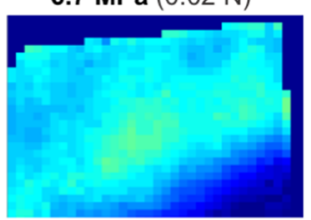

101.3 $\mathrm{MPa}(0.35 \mathrm{~N})$

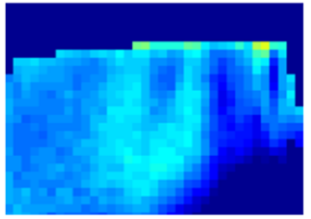

$15.8 \mathrm{MPa}(0.05 \mathrm{~N})$

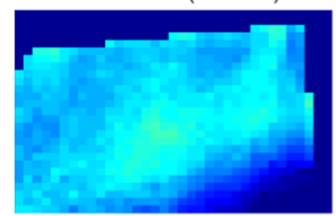

195.6 MPa $(0.67 \mathrm{~N})$

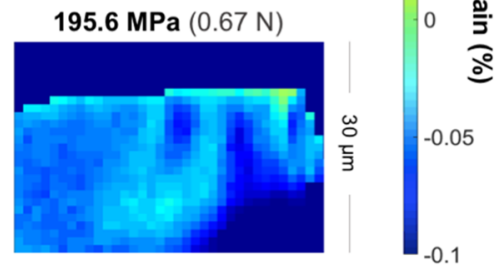

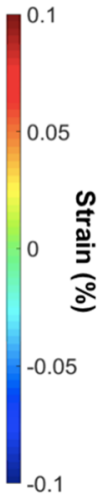

.

Figure 3. (A) As-measured WAXS pattern of the main pillar. (B) Strain maps relative to the iron oxide NPs' crystalline lattice for increasing applied compressive stresses (step size: $2 \mu \mathrm{m}$ horizontally and $1 \mu \mathrm{m}$ vertically). The reference zero-strain configuration was the preloading scan of the micropillar, based on which the strains in part B were calculated. The apparently different scaling in the different plots of part B is due to the inclination of the WAXS detector with respect to the beam.

A

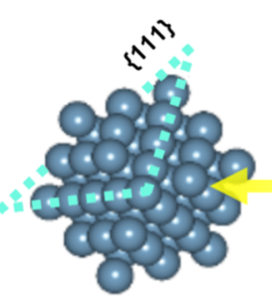

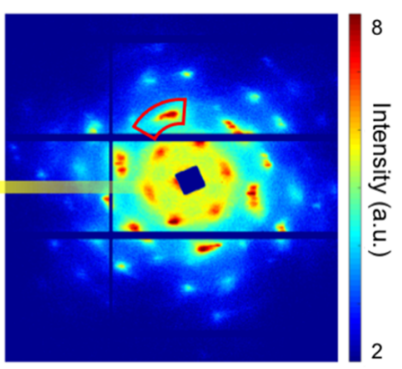

B

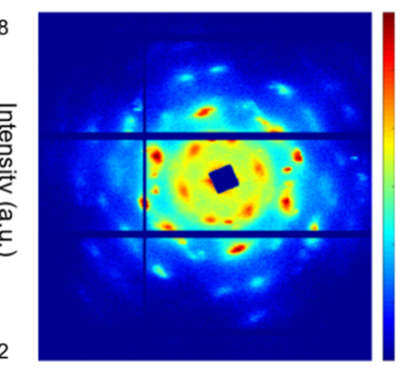

C

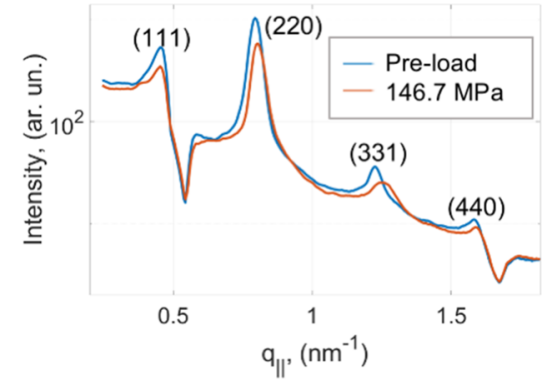

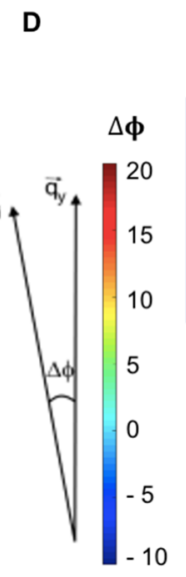

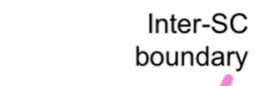

boundary
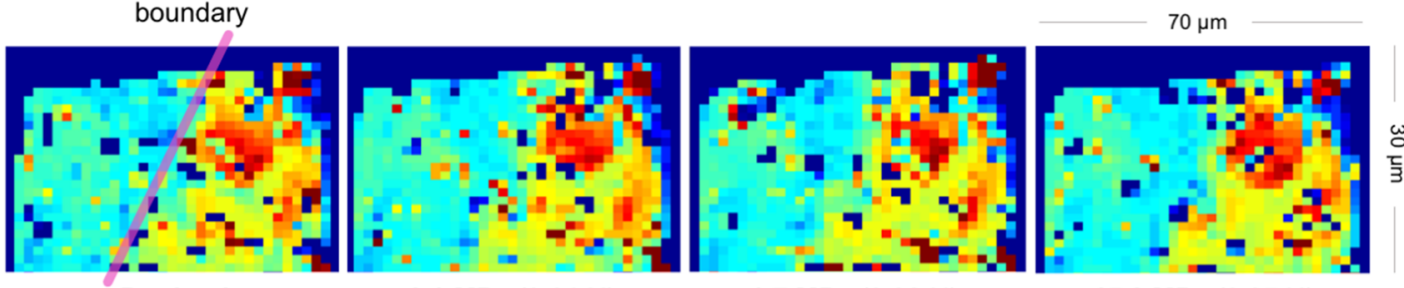

$6.7 \mathrm{MPa}(0.02 \mathrm{~N})$

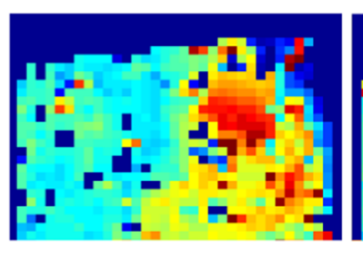

2.6 MPa $(0.01 \mathrm{~N})$

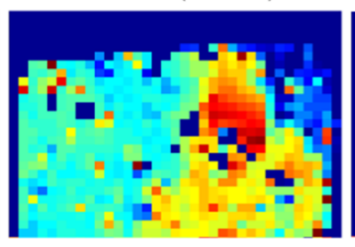

101.3 MPa (0.35 N)

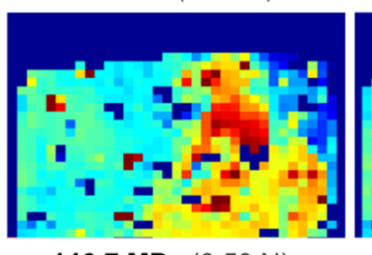

15.8 $\mathrm{MPa}(0.05 \mathrm{~N})$

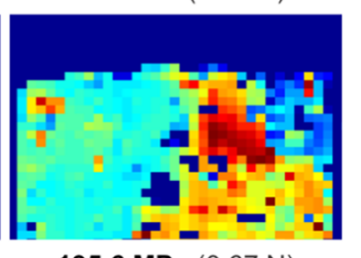

195.6 MPa $(0.67 \mathrm{~N})$

Figure 4. (A) Representative SAXS pattern of undeformed pillar with reconstruction of corresponding FCC unit cell in real space (type $\{111\}$ plane highlighted and azimuthal integration also schematized in the SAXS spectrum). (B) SAXS pattern of same point under $146.7 \mathrm{MPa}(0.50 \mathrm{~N})$ load. (C) Integrated SAXS spectra of the location shown in parts A and B before loading and at $146.7 \mathrm{MPa}$ (0.50 N) load. (D) Supercrystalline orientations (deg) mapping at increasing loads, as derived through azimuthal integration of the highlighted peak in the SAXS spectra (marked in part A). An intersupercrystalline boundary is marked in the preload mapping.

bottom-right corner of the pillar, are due to an impurity initially sitting on the micropillar's surface (see Figure S2), leading to a load concentration. The strains experienced by the NPs during uniaxial compression increase with loading, up to $0.1 \%$ values. Slight strain gradients along the direction parallel to the applied load suggest gradual load propagation from the pillar's edges toward the center.
Importantly, no $\mu \mathrm{m}$-scale load percolation path is detected, indicating a uniform load distribution on the NPs through the cross-linked organic phase. Nonuniformities along the applied load direction, hinting at preferential load-carrying paths, appear only at higher loads, approaching the onset of the block slip detected via optical microscope (see Figure 2). This suggests that after severe slip, broken covalent bonds in the 
organic phase allow direct contacts between inorganic surfaces. If some NPs come into direct contact prior to the block slip, these local contacts are in low number and uniformly scattered.

The maximum strains detected in the NPs, $0.1 \%$, are 1 order of magnitude lower than the ones measured for the overall supercrystalline nanocomposites (a few \%), implying that the organic phase undergoes strains in the order of $10 \%$ (see Supporting Information section 4 and Figure S6). The measured intra-NP strains also correlate well with the stresses at which they occur, $\sim 190 \mathrm{MPa}$, through Hooke's law. The elastic modulus for iron oxide is reported to span a relatively broad range of values. ${ }^{49,50}$ Due to the nanocrystalline nature of the NPs, here we consider the range relative to single crystals (163-175 GPa), keeping in mind that nanocrystals sometimes feature higher elastic moduli than their bulk counterparts. ${ }^{51}$ The supercrystalline nanocomposites are of the face-centered cubic (FCC) type ${ }^{19,22}$ (therefore each NP has 12 nearest neighbors), and typically polysupercrystalline. This arrangement allows considering the overall material mechanically quasi-isotropic, as FEM simulations have confirmed. ${ }^{22}$ FEM analyses also reveal that under uniaxial pressing conditions, stress concentrations arise at most inter-NP contacts, intensifying the applied stress by up to a factor of 3 , while adjacent to the interstitial sites and at the interfaces aligned along the pressing load such concentrations are absent (see Supporting Information section 5 and Figure S7). Since each $\mathrm{NP}$ is randomly oriented within the superlattice, the strains depicted in Figure 3B can be considered representative average strains of the intra-NP crystalline lattice. Such a load distribution within the inorganic nanoparticles confirms the important role of the organic phase (anchored to the nanoparticles, confined into sub-nm interfaces, and crosslinked) toward the material's load carrying ability. Previous works had indeed already underlined how the mechanical behavior of supercrystals correlates better with polymer-matrix nanocomposites than with single phase colloidal crystals. ${ }^{14}$ Even though in those cases the organic ligands were not crosslinked, this phase was described as "cohesive organic network". Cross-linking stiffens this network, reinforcing the applicability of this model.

The remarkable resistance to deformation of the cross-linked oleic acid phase is confirmed via SAXS. An example of SAXS pattern with the typical 6-fold symmetry of FCC arrangements (in agreement with previous analyse ${ }^{18-20}$ ) is shown in Figure $4 \mathrm{~A}$, with a representative reconstruction of the corresponding NP superstructure in the real space for preloading conditions. Before compression, an average superlattice parameter $a=23.1$ $\pm 0.6 \mathrm{~nm}$ is measured for the micropillar. Given the NP radius $R=7.9 \mathrm{~nm}$, an interparticle distance (along the tightly packed $\langle 110\rangle$ directions) ID $=a / \sqrt{2}-2 R=0.5 \mathrm{~nm}$ is found, implying a high confinement of the oleic acid chains $(\sim 2 \mathrm{~nm}$ long at full extension). ${ }^{52}$ The annealing does induce not only cross-linking but also an overall superlattice shrinkage, typically resulting in a $5 \%$ reduction in $a .{ }^{18,19}$ Such a small ID value, together with cross-linking, prevents the NPs from further approaching each other. The high stiffness of the cross-linked organic phase has also been confirmed with a FEM study that has resulted in elastic modulus $E=13 \mathrm{GPa}$ for the oleic acid phase confined in a $0.6 \mathrm{~nm} \mathrm{ID}, 22$ a value much higher than the ones estimated for non-cross-linked supercrystals. ${ }^{14}$ Also in contrast to previous in situ compression studies on supercrystals, ${ }^{39}$ we did not observe improved superlattice ordering, ligand extrusion, or NP sintering. These differences are due not only to the loading conditions but also to the stiff network of covalently bonded ligands.

Figure 4B and Figure 4C show the effect of loading at 146.7 $\mathrm{MPa}(0.50 \mathrm{~N})$ on the SAXS spectrum and intensity plot of the same area considered in preloading conditions (Figure 4A). The FCC arrangement is likely slightly distorted but not disrupted, and a shift of the superlattice reflections toward higher scattering vector values $(q)$ is observed, implying a reduction in distances among supercrystalline planes $(d \sim 2 \pi)$ $q)$. These shrinkage values change depending on the considered reflection, and they vary throughout the micropillar. Tensile strains are also locally detected, relatable to Poisson effects, even though compressive strains prevail. The strain distribution scatter is expected in such large micropillars with no predetermined orientation of the superlattice with respect to the applied load, but trends can be identified. For the case of $101.3 \mathrm{MPa}(0.35 \mathrm{~N}$, prior to the block slip), by averaging interplanar shrinkages along the $\{111\},\{220\}$, and $\{331\}$ reflections in different micropillar locations (Supporting Information section 6), an average compressive strain of $1.4 \%$ is estimated (the FCC structure guarantees elastic quasiisotropy, as confirmed by molecular dynamics simulations ${ }^{41}$ ). This is compatible with the preservation of an organic-filled gap between NPs $\left(\mathrm{ID}_{\mathrm{f}} \sim 0.3 \mathrm{~nm}\right.$; see Supporting Information section 4). It is also slightly lower than the strain measured by the microcompression setup at this load (1.6\%, Figure S3), suggesting that the pop-in-like effects are associated with defects such as the organic-rich clusters shown in Figure S4 instead of intrasuperlattice deformations.

SAXS reveals additional superlattice deformation mechanisms. The loading configuration is particularly suitable for material slip, which has been observed in the form of sliding of a supercrystalline block, likely along tightly packed superlattice planes of the $\{111\}$ family (see Figures 2 and $4 A$ ). In Figure $4 \mathrm{C}$, a decrease in the intensity of the superlattice reflections is observed, accompanied by a peak broadening, indicating degradation of the supercrystalline structure. In Figure 4B one can also identify azimuthal peak splitting at the $\{111\}$ planes level, an indication for twinning phenomena (see Supporting Information section 7 and Figure S8). ${ }^{53,54}$ The analysis of the SAXS peaks variations in the azimuthal direction reveals how the FCC superlattice is oriented within the micropillar at the various compression stages; see Figure 4D. The micropillar has a predominantly bisupercrystalline structure, with a $10^{\circ}$ mismatch among the two domains ("grains") separated by a boundary oriented $\sim 60^{\circ}$ with respect to the applied load. Within the domain on the right side of the micropillar, an additional variation of about $5^{\circ}$ is detected. Its contours are not sharp, indicating a gradual superlattice rotation, also detected via SEM (Figure S4). These different supercrystalline domains can also be identified in the postmortem pillar micrographs (Figure S4). The domain on the right-hand-side of Figure 4 is the one undergoing the most severe reorientations (see load concentration shown via WAXS, Figure 3). A region with a $20^{\circ}$ orientation mismatch appears and gradually widens, corresponding to the area where slip occurred, which then does not occur on a single gliding plane. The bottom-right region of the pillar also shows superlattice rotations of $\sim 5^{\circ}$.

Through the combination of uniaxial pressing and both wide- and small-angle X-ray scattering on a cross-linkingstrengthened supercrystalline nanocomposite, it has then been shown that the remarkable resistance of supercrystals to 
compression goes beyond hydrostatic pressing. The crosslinked organic phase leads to resistance of the material to strains and homogeneously transmits the applied loads to the NPs. No microscale load percolation paths were observed, reinforcing the assumption of the cross-linked organic network playing a key strengthening and stiffening role. WAXS-detected strains within the NPs of the order of $\sim 0.1 \%$ and overall SAXSdetected mesoscale strains in the superlattice of $\sim 1-3 \%$ imply that the organic phase undergoes larger deformations $(\sim 10 \%)$. The nanocomposites also accommodate loading via superlattice rotation and defects formation.

\section{ASSOCIATED CONTENT}

\section{(s) Supporting Information}

The Supporting Information is available free of charge at https://pubs.acs.org/doi/10.1021/acs.nanolett.0c05041.

Sections 1-7, Figures S1-S8, Tables S1 and S2, and methods (PDF)

\section{AUTHOR INFORMATION}

\section{Corresponding Author}

Diletta Giuntini - Institute of Advanced Ceramics, Hamburg University of Technology, 21073 Hamburg, Germany; (i) orcid.org/0000-0003-3338-6432;

Email: diletta.giuntini@tuhh.de,d.giuntini@tue.nl

\section{Authors}

Anton Davydok - Institute of Materials Physics, HelmholtzZentrum Geesthacht, 21502 Geesthacht, Germany

Malte Blankenburg - Institute of Materials Physics, Helmholtz-Zentrum Geesthacht, 21502 Geesthacht, Germany

Berta Domènech - Institute of Advanced Ceramics, Hamburg University of Technology, 21073 Hamburg, Germany; (1) orcid.org/0000-0003-2042-4428

Büsra Bor - Institute of Advanced Ceramics, Hamburg University of Technology, 21073 Hamburg, Germany

Mingjing Li - Institute of Material Systems Modeling, Helmholtz-Zentrum Geesthacht, 21502 Geesthacht, Germany

Ingo Scheider - Institute of Material Systems Modeling, Helmholtz-Zentrum Geesthacht, 21502 Geesthacht, Germany

Christina Krywka - Institute of Materials Physics, HelmholtzZentrum Geesthacht, 21502 Geesthacht, Germany

Martin Müller - Institute of Materials Physics, Helmholtz-

Zentrum Geesthacht, 21502 Geesthacht, Germany

Gerold A. Schneider - Institute of Advanced Ceramics, Hamburg University of Technology, 21073 Hamburg, Germany

Complete contact information is available at:

https://pubs.acs.org/10.1021/acs.nanolett.0c05041

\section{Author Contributions}

D.G., A.D., B.D., and G.A.S. conceived and designed the study. D.G., A.D., M.B., B.D., and B.B. conducted the study. D.G., A.D., and M.B. analyzed and interpreted the data. M.L. and I.S. conducted the supporting numerical study. C.K., M.M., and G.A.S. supervised the work. D.G. wrote the first manuscript draft. The manuscript was finalized through contributions of all authors. All authors have given approval to the final version of the manuscript.

\section{Funding}

The authors gratefully acknowledge financial support from the Deutsche Forschungsgemeinschaft (DFG, German Research
Foundation), Projektnummer 192346071-SFB 986. D.G. gratefully acknowledges the support from the Alexander von Humboldt Foundation. B.B. gratefully acknowledges the support from the Ministry of National Education of the Republic of Turkey. The authors are very grateful to Dr. Martin Ritter for his valuable input and support during sample microstructuring.

\section{Notes}

The authors declare no competing financial interest.

\section{REFERENCES}

(1) Talapin, D. V.; Lee, J.-S.; Kovalenko, M. V.; Shevchenko, E. V. Prospects of colloidal nanocrystals for electronic and optoelectronic applications. Chem. Rev. 2010, 110 (1), 389-458.

(2) Tachikawa, T.; Majima, T. Metal oxide mesocrystals with tailored structures and properties for energy conversion and storage applications. NPG Asia Mater. 2014, 6 (5), No. e100.

(3) Sturm, E.; Cölfen, H. Mesocrystals: past, present, future. Crystals 2017, 7 (7), 207.

(4) Schliehe, C.; Juarez, B. H.; Pelletier, M.; Jander, S.; Greshnykh, D.; Nagel, M.; Meyer, A.; Foerster, S.; Kornowski, A.; Klinke, C.; Weller, $\mathrm{H}$. Ultrathin $\mathrm{PbS}$ sheets by two-dimensional oriented attachment. Science 2010, 329 (5991), 550-553.

(5) Pileni, M. P. Supracrystals of inorganic nanocrystals. Acc. Chem. Res. 2008, 41, 1799-1809.

(6) Pileni, M. P. Nanocrystal Self-Assemblies. J. Phys. Chem. B 2001, 105 (17), 3358-3371.

(7) Murray, C. B.; Kagan, C. R.; Bawendi, M. G. Synthesis and Characterization of Monodisperse Nanocrystals and Close-Packed Nanocrystal Assemblies. Annu. Rev. Mater. Sci. 2000, 30 (1), 545610.

(8) Glotzer, S. C.; Solomon, M. J.; Kotov, N. A. Self-assembly: From microscale to nanoscale colloids. AIChE J. 2004, 50 (12), 2978-2985.

(9) Eder, M.; Amini, S.; Fratzl, P. Biological composites-complex structures for functional diversity. Science 2018, 362 (6414), 543547.

(10) Begley, M. R.; Gianola, D. S.; Ray, T. R. Bridging functional nanocomposites to robust macroscale devices. Science 2019, 364 (6447), eaav4299.

(11) Mueggenburg, K. E.; Lin, X.-M.; Goldsmith, R. H.; Jaeger, H. M. Elastic membranes of close-packed nanoparticle arrays. Nat. Mater. 2007, 6 (9), 656-660.

(12) He, J.; Kanjanaboos, P.; Frazer, N. L.; Weis, A.; Lin, X.-M.; Jaeger, H. M. Fabrication and mechanical properties of large-scale freestanding nanoparticle membranes. Small 2010, 6 (13), 14491456.

(13) Yan, C.; Arfaoui, I.; Goubet, N.; Pileni, M.-P. Soft Supracrystals of Au Nanocrystals with Tunable Mechanical Properties. Adv. Funct. Mater. 2013, 23 (18), 2315-2321.

(14) Podsiadlo, P.; Krylova, G.; Lee, B.; Critchley, K.; Gosztola, D. J.; Talapin, D. V.; Ashby, P. D.; Shevchenko, E. V. The role of order, nanocrystal size, and capping ligands in the collective mechanical response of three-dimensional nanocrystal solids. J. Am. Chem. Soc. 2010, 132 (26), 8953-8960.

(15) Tam, E.; Podsiadlo, P.; Shevchenko, E.; Ogletree, D. F.; Delplancke-Ogletree, M.-P.; Ashby, P. D. Mechanical properties of face-centered cubic supercrystals of nanocrystals. Nano Lett. 2010, 10 (7), 2363-2367.

(16) Pileni, M.-P. Mechanical properties of supracrystals. EPL 2017, 119 (3), 37002.

(17) Gu, X. W. Mechanical Properties of Architected Nanomaterials Made from Organic-Inorganic Nanocrystals. JOM 2018, 70 (10), 2205-2217.

(18) Dreyer, A.; Feld, A.; Kornowski, A.; Yilmaz, E. D.; Noei, H.; Meyer, A.; Krekeler, T.; Jiao, C.; Stierle, A.; Abetz, V.; Weller, H.; Schneider, G. A. Organically linked iron oxide nanoparticle supercrystals with exceptional isotropic mechanical properties. Nat. Mater. 2016, 15 (5), 522-528. 
(19) Bor, B.; Giuntini, D.; Domènech, B.; Swain, M. V.; Schneider, G. A. Nanoindentation-based study of the mechanical behavior of bulk supercrystalline ceramic-organic nanocomposites. J. Eur. Ceram. Soc. 2019, 39 (10), 3247-3256.

(20) Domènech, B.; Plunkett, A.; Kampferbeck, M.; Blankenburg, M.; Bor, B.; Giuntini, D.; Krekeler, T.; Wagstaffe, M.; Noei, H.; Stierle, A.; Ritter, M.; Müller, M.; Vossmeyer, T.; Weller, H.; Schneider, G. A. Modulating the mechanical properties of supercrystalline nanocomposite materials via solvent-ligand interactions. Langmuir 2019, 35, 13893-13903.

(21) Giuntini, D.; Torresani, E.; Chan, K. T.; Blankenburg, M.; Saviot, L.; Bor, B.; Domènech, B.; Shachar, M.; Müller, M.; Olevsky, E. A.; Garay, J. E.; Schneider, G. A. Iron oxide-based nanostructured ceramics with tailored magnetic and mechanical properties. Nanoscale Adv. 2019, 1 (8), 3139-3150.

(22) Li, M.; Scheider, I.; Bor, B.; Domènech, B.; Schneider, G. A.; Giuntini, D. Ultra-thin and ultra-strong organic interphase in nanocomposites with supercrystalline particle arrangement. Compos. Sci. Technol. 2020, 198, 108283.

(23) Bor, B.; Heilmann, L.; Domènech, B.; Kampferbeck, M.; Vossmeyer, T.; Weller, H.; Schneider, G. A.; Giuntini, D. Mapping the Mechanical Properties of Hierarchical Supercrystalline CeramicOrganic Nanocomposites. Molecules 2020, 25 (20), 4790.

(24) Domènech, B.; Kampferbeck, M.; Larsson, E.; Krekeler, T.; Bor, B.; Giuntini, D.; Blankenburg, M.; Ritter, M.; Müller, M.; Vossmeyer, T.; Weller, H.; Schneider, G. A. Hierarchical supercrystalline nanocomposites through the self-assembly of organicallymodified ceramic nanoparticles. Sci. Rep. 2019, 9 (1), 3435.

(25) Domènech, B.; Tan, A. T. L.; Jelitto, H.; Zegarra Berodt, E.; Blankenburg, M.; Focke, O.; Cann, J.; Cem Tasan, C.; Colombi Ciacchi, L.; Müller, M.; Furlan, K. P.; John Hart, A.; Schneider, G. A. Strong Macroscale Supercrystalline Structures by 3D Printing Combined with Self-Assembly of Ceramic Functionalized Nanoparticles. Adv. Eng. Mater. 2020, 22 (7), 2000352.

(26) Wang, Z.; Singaravelu, A. S. S.; Dai, R.; Nian, Q.; Chawla, N.; Wang, R. Y. Ligand Crosslinking Boosts Thermal Transport in Colloidal Nanocrystal Solids. Angew. Chem., Int. Ed. 2020, 59 (24), 9556-9563.

(27) Chen; Herhold; Johnson; Alivisatos. Size Dependence of Structural Metastability in Semiconductor Nanocrystals. Science 1997, 276 (5311), 398-401.

(28) Boles, M. A.; Engel, M.; Talapin, D. V. Self-Assembly of Colloidal Nanocrystals. Chem. Rev. 2016, 116 (18), 11220-11289.

(29) Banerjee, S.; Jia, S.; Kim, D. I.; Robinson, R. D.; Kysar, J. W.; Bevk, J.; Herman, I. P. Raman microprobe analysis of elastic strain and fracture in electrophoretically deposited CdSe nanocrystal films. Nano Lett. 2006, 6 (2), 175-180.

(30) Lee, B.; Podsiadlo, P.; Rupich, S.; Talapin, D. V.; Rajh, T.; Shevchenko, E. V. Comparison of structural behavior of nanocrystals in randomly packed films and long-range ordered superlattices by time-resolved small angle X-ray scattering. J. Am. Chem. Soc. 2009, 131 (45), 16386-16388.

(31) Wu, H.; Bai, F.; Sun, Z.; Haddad, R. E.; Boye, D. M.; Wang, Z.; Huang, J. Y.; Fan, H. Nanostructured gold architectures formed through high pressure-driven sintering of spherical nanoparticle arrays. J. Am. Chem. Soc. 2010, 132 (37), 12826-12828.

(32) Wu, H.; Bai, F.; Sun, Z.; Haddad, R. E.; Boye, D. M.; Wang, Z.; Fan, H. Pressure-driven assembly of spherical nanoparticles and formation of 1D-nanostructure arrays. Angew. Chem., Int. Ed. 2010, 49 (45), 8431-8434.

(33) Wang, Z.; Schliehe, C.; Wang, T.; Nagaoka, Y.; Cao, Y. C.; Bassett, W. A.; Wu, H.; Fan, H.; Weller, H. Deviatoric stress driven formation of large single-crystal $\mathrm{PbS}$ nanosheet from nanoparticles and in situ monitoring of oriented attachment. J. Am. Chem. Soc. 2011, 133, 14484-14487.

(34) Bian, K.; Wang, Z.; Hanrath, T. Comparing the structural stability of $\mathrm{PbS}$ nanocrystals assembled in fcc and bcc superlattice allotropes. J. Am. Chem. Soc. 2012, 134 (26), 10787-10790.
(35) Li, B.; Wen, X.; Li, R.; Wang, Z.; Clem, P. G.; Fan, H. Stressinduced phase transformation and optical coupling of silver nanoparticle superlattices into mechanically stable nanowires. Nat. Commun. 2014, 5, 4179.

(36) Podsiadlo, P.; Lee, B.; Prakapenka, V. B.; Krylova, G. V.; Schaller, R. D.; Demortière, A.; Shevchenko, E. V. High-pressure structural stability and elasticity of supercrystals self-assembled from nanocrystals. Nano Lett. 2011, 11 (2), 579-588.

(37) Wu, H.; Wang, Z.; Fan, H. Stress-induced nanoparticle crystallization. J. Am. Chem. Soc. 2014, 136 (21), 7634-7636.

(38) Nagaoka, Y.; Hills-Kimball, K.; Tan, R.; Li, R.; Wang, Z.; Chen, O. Nanocube Superlattices of Cesium Lead Bromide Perovskites and Pressure-Induced Phase Transformations at Atomic and Mesoscale Levels. Adv. Mater. 2017, 29 (18), 1606666.

(39) Bai, F.; Bian, K.; Huang, X.; Wang, Z.; Fan, H. Pressure Induced Nanoparticle Phase Behavior, Property, and Applications. Chem. Rev. 2019, 119 (12), 7673-7717.

(40) Li, W.; Fan, H.; Li, J. Deviatoric stress-driven fusion of nanoparticle superlattices. Nano Lett. 2014, 14 (9), 4951-4958.

(41) Patra, T. K.; Chan, H.; Podsiadlo, P.; Shevchenko, E. V.; Sankaranarayanan, S. K. R. S.; Narayanan, B. Ligand dynamics control structure, elasticity, and high-pressure behavior of nanoparticle superlattices. Nanoscale 2019, 11 (22), 10655-10666.

(42) Zeilinger, A.; Todt, J.; Krywka, C.; Müller, M.; Ecker, W.; Sartory, B.; Meindlhumer, M.; Stefenelli, M.; Daniel, R.; Mitterer, C.; Keckes, J. In-situ Observation of Cross-Sectional Microstructural Changes and Stress Distributions in Fracturing TiN Thin Film during Nanoindentation. Sci. Rep. 2016, 6, 22670.

(43) Zhang, H.; Schuster, B. E.; Wei, Q.; Ramesh, K. T. The design of accurate micro-compression experiments. Scr. Mater. 2006, 54 (2), $181-186$.

(44) Danzer, R.; Lube, T.; Supancic, P.; Damani, R. Fracture of Ceramics. Adv. Eng. Mater. 2008, 10 (4), 275-298.

(45) Choi, I.-s.; Gan, Y.; Kaufmann, D.; Kraft, O.; Schwaiger, R. Measurement of Young's modulus of anisotropic materials using microcompression testing. J. Mater. Res. 2012, 27 (21), 2752-2759.

(46) Lee, C. J.; Huang, J. C.; Nieh, T. G. Sample size effect and microcompression of Mg65Cu25Gd10 metallic glass. Appl. Phys. Lett. 2007, 91 (16), 161913.

(47) Meyers, M. A.; Chawla, K. K. Mechanical Behavior of Materials, 2nd ed.; Cambridge University Press: Cambridge, U.K., 2009.

(48) Cornell, R. M.; Schwertmann, U. The Iron Oxides; Wiley-VCH Verlag GmbH \& Co. KGaA: Weinheim, Germany, 2003.

(49) Chicot, D.; Mendoza, J.; Zaoui, A.; Louis, G.; Lepingle, V.; Roudet, F.; Lesage, J. Mechanical properties of magnetite (Fe3O4), hematite $(\alpha-\mathrm{Fe} 2 \mathrm{O} 3)$ and goethite $(\alpha-\mathrm{FeO} \cdot \mathrm{OH})$ by instrumented indentation and molecular dynamics analysis. Mater. Chem. Phys. 2011, 129 (3), 862-870.

(50) Reichmann, H. J.; Jacobsen, S. D. High-pressure elasticity of a natural magnetite crystal. Am. Mineral. 2004, 89 (7), 1061-1066.

(51) Guo, D.; Xie, G.; Luo, J. Mechanical properties of nanoparticles: basics and applications. J. Phys. D: Appl. Phys. 2014, 47 (1), 013001.

(52) Gunstone, F. D.; Harwood, J. L.; Dijkstra, A. J. The Lipid Handbook, 3rd ed.; CRC Press: Boca Raton, FL, 2007.

(53) Taheri, S. M.; Rosenfeldt, S.; Fischer, S.; Bösecke, P.; Narayanan, T.; Lindner, P.; Förster, S. Shear-induced macroscopic "Siamese" twins in soft colloidal crystals. Soft Matter 2013, 9 (35), 8464.

(54) Pietsch, U.; Holy, V.; Baumbach, T. High-Resolution X-Ray Scattering: From Thin Films to Lateral Nanostructures, 2nd ed.; Springer: New York, 2004. 